\title{
Autoantibodies against myelin antigens in patients with neuromyelitis optica
}

\author{
Kota Moriguchi $^{1}$, Katsuichi Miyamoto ${ }^{2^{*}}$, Juri Ichihashi ${ }^{2}$, Susumu Kusunoki ${ }^{2}$ \\ ${ }^{1}$ Department of Internal Medicine 3, National Defense Medical College, Tokorozawa, Japan \\ ${ }^{2}$ Department of Neurology, Kinki University School of Medicine, Osaka, Japan; \\ *Corresponding Author:miyamoto@med.kindai.ac.jp
}

Received 15 March 2013; revised 14 April 2013; accepted 28 April 2013

Copyright (C) 2013 Kota Moriguchi et al. This is an open access article distributed under the Creative Commons Attribution License, which permits unrestricted use, distribution, and reproduction in any medium, provided the original work is properly cited.

\begin{abstract}
In this study, we investigated the clinical relevance of anti-myelin antibodies in patients with neuromyelitis optica (NMO); titers of antibodies against myelin oligodendrocyte glycoproteins, proteolipid proteins and myelin basic proteins were measured in the sera of patients with NMO and compared to healthy controls, as well as to patients with other diseases. The frequency of presence of anti-myelin antibodies in patients with NMO was significantly higher than that in healthy and diseased controls. The expanded disability status scale scores correlated with the titers of the anti-myelin antibodies. Patients with anti-myelin antibody exhibited other autoantibodies significantly more frequently than patients without the antibody. Anti-myelin antibodies may be useful markers for predicting severe clinical courses in patients with NMO.
\end{abstract}

Keywords: Neuromyelitis Optica; Anti-Myelin Antibody; Myelin Oligodendrocyte Glycoprotein; Proteolipid Protein; Myelin Basic Protein

\section{INTRODUCTION}

Neuromyelitis optica (NMO) is an idiopathic and severe demyelinating, inflammatory disease that attacks the optic nerve and spinal cord. While this rare disorder resembles multiple sclerosis (MS) in several ways, recent studies have demonstrated that NMO can be clearly distinguished through its clinical course, prognosis, pathological features, and etiology. Patients with NMO often have a poor prognosis and relapsing clinical course. More than half of them develop severe visual loss or an inability to ambulate without assistance within a few years of disease onset [1]. The pathological features of
NMO are characterized by immunoglobulin deposition, complement activation, vascular hyalinization and granulocyte infiltration of active lesions [2]. Based on the discovery of an autoantibody in patients with $\mathrm{NMO}$, which is directed against the water channel aquaporin-4 (AQP4), NMO is now considered as an antibody-mediated autoimmune disease [3].

Proposed targets of the immune response in inflammatory demyelinating diseases of the central nervous system include myelin antigens such as myelin oligodendrocyte glycoprotein (MOG), proteolipid protein (PLP), and myelin basic protein (MBP) [4]. It has been demonstrated that antibodies against MOG can cause demyelination in vitro [5] and in animal models [6]. While many studies have been performed to investigate anti-myelin antibodies in patients with MS, the pathogenic or predicttive role of these antibodies remains uncertain [7,8]. Few investigations have been conducted in order to evaluate the clinical relevance of anti-myelin antibodies in NMO [9]. Herein, we report on a retrospective case-control study of 17 NMO patients with anti-AQP4 antibodies in order to evaluate the clinical relevance of anti-myelin antibodies in these patients.

\section{METHODS}

\subsection{Patients and Controls}

Serum samples from 17 patients with NMO spectrum disorders, including $\mathrm{NMO}$ and at-risk syndromes that fulfilled the criteria proposed by Wingerchuk [10], were investigated. The serum samples were kept frozen at $-80^{\circ} \mathrm{C}$ until time of use. All patients with NMO had high titers of the anti-AQP4 antibody. We also investigated samples from 30 healthy controls, as well as 63 controls with the following diseases: MS $(n=21)$, Guillain-Barré syndrome $(\mathrm{n}=16)$, chronic inflammatory demyelinating polyradiculopathy $(\mathrm{n}=4)$, Miller Fisher syndrome $(\mathrm{n}=$ $2)$, cerebral infarction $(n=6)$ and other neurodegenera 
tive diseases $(\mathrm{n}=14)$. Informed consent was obtained, and the study was approved by the internal review board of Kinki University Faculty of Medicine.

\subsection{ELISA for Antibodies against Myelin Antigens}

The presence of serum antibodies against myelin antigens was investigated with enzyme-linked immunosorbent assays (ELISA), as previously described [7]. Microtiter plates were coated with $0.5 \mu \mathrm{g}$ of MOG (35 - 55), PLP (183 - 199), or MBP (83 - 99) (Toray Research Center, Inc., Tokyo, Japan). These peptides were extracellular domains of the naive antigens and were considered epitopes against autoantibodies in demyelinating diseases of the central nervous system [11,12]. An uncoated well was used as a control. After incubation with $1 \%$ bovine serum albumin (BSA) in phosphate-buffered saline (PBS), the serum was diluted to $1: 40$ with $1 \%$ BSA in PBS and added to each well; the plate was incubated at room temperature for $2 \mathrm{~h}$. After washing with PBS, peroxidase-conjugated goat anti-human IgG antibody (Jackson ImmunoResearch Laboratories, Inc., West Grove, PA, USA), which was diluted 1:10000 with 1\% BSA in PBS, or peroxidase-conjugated goat anti-human IgM (MP Biomedicals LLC, Irvine, CA, USA), which was diluted 1:200 with the same solution, was added to each well. After incubation, the wells were washed and orthophenylenediamine dihydrochloride $(40 \mathrm{mg} / \mathrm{dL}$ of phosphate-citrate buffer, $\mathrm{pH}$ 5.0) was used to obtain a color reaction. The reaction was stopped by the addition of $8 \mathrm{~N}$ $\mathrm{H}_{2} \mathrm{SO}_{4}$, after which the optical density (OD) of each well was read at $490 \mathrm{~nm}$ using an ELISA reader (Model 550, Bio-Rad, Hercules, California, USA). Each OD value was corrected by subtracting the OD value of the control well that had been similarly processed. The ELISAs were repeated 3 times, and the mean OD of the 3 experiments was calculated. Sera with corrected OD values that were greater than $+3 \mathrm{SD}$ of the normal control values were considered positive.

\subsection{Statistical Analysis}

The differences in the populations were tested by the $\mathrm{X}^{2}$ test for independence. The $\mathrm{F}$ test was used to assess whether the distributions of the data variables were equal among the groups. The two-sample $t$ test and the Welch's $t$ test were used to compare the differences between groups (anti-myelin antibody-positive and anti-myelin antibody-negative). The Pearson product moment correlation coefficient was used to assess the correlations among the data variables. A two-tailed $P$ value of less than 0.05 was considered statistically significant.

\section{RESULTS}

\subsection{Frequencies of Anti-Myelin Antibodies}

The mean +3 SD values of the corrected OD values of the healthy controls were as follows: 0.131 for the antiMOG IgM antibody; 0.089 for the anti-PLP IgM antibody; 0.086 for the anti-MBP IgM antibody, 0.041 for the anti-MOG IgG antibody, 0.055 for the anti-PLP IgG antibody, and 0.064 for the anti-MBP IgG antibody. None of the 30 healthy controls or the 63 diseased controls was positive for anti-myelin antibodies. No significant differences in frequency were found among normal and disease controls. In comparison, 5 of the 17 patients with NMO (29\%) exhibited at least one of the anti-myelin antibodies $\left(P<0.0001\right.$ in a $\mathrm{X}^{2}$ test $)$. A summary of the patients with antibodies to myelin antigens is shown in Table 1.

\subsection{Clinical Features of Patients with NMO and Anti-Myelin Antibodies}

Patients with NMO were divided into anti-myelin antibody-positive and anti-myelin antibody-negative groups. The clinical features of the patients in each group are presented in Table 2. Cerebrospinal fluid (CSF) samples were obtained in order to examine the cell count; the content of IgG was examined on the same day as the serum. Scores on the expanded disability status scale (EDSS) were assessed when the patients' conditions were at their worst.

Since the F test used to test the parameters of the CSF cell count and EDSS did not show an equal distribution of the data variables among the groups, a Welch's $t$ test was employed. Since the CSF IgG and attack frequency parameters exhibited an equal distribution, a two-sample $t$ test was used.

Table 1. Summary of patients with neuromyelitis optica (NMO) and antibodies to myelin antigens.

\begin{tabular}{ccccc}
\hline Patient & Age $(\mathrm{y}) /$ sex & Disease & EDSS & \multicolumn{2}{c}{ IgM antibodies } \\
\hline 1 & $20 / \mathrm{F}$ & NMO & 6 & MOG, PLP \\
2 & $60 / \mathrm{F}$ & NMO overlapping APS & 7 & MOG, PLP, MBP \\
3 & $52 / \mathrm{F}$ & NMO & 8.5 & negative \\
4 & $48 / \mathrm{F}$ & NMO & 8 & negative \\
5 & $32 / \mathrm{F}$ & NMOSD & 4.5 & PLP, MBP \\
\hline
\end{tabular}

APS, anti-phospholipid antibody syndrome; EDSS, expanded disability status scale; MBP, myelin basic protein; MOG, myelin oligodendrocyte glycoprotein; NMOSD, spectrum disease; PLP, proteolipid protein. 
Table 2. Clinical features of patients with NMO with and without anti-myelin antibodies.

\begin{tabular}{|c|c|c|}
\hline Clinical features & Positive $(\mathrm{n}=5)$ & Negative $(\mathrm{n}=12)$ \\
\hline Age (years) ${ }^{*}$ & $42.0 \pm 14$ & $53 \pm 17$ \\
\hline Males: females & $0: 5$ & $1: 11$ \\
\hline \multicolumn{3}{|l|}{ Brain magnetic resonance imaging } \\
\hline Number of patients with abnormalities & $4(80 \%)$ & $8(67 \%)$ \\
\hline Location & cerebrum, brainstem & cerebrum, brainstem, pons \\
\hline Optic neuritis & $2(40 \%)$ & $4(33 \%)$ \\
\hline Long spinal cord lesion & $2(40 \%)$ & $8(67 \%)$ \\
\hline Cerebrospinal fluid (CSF) cell count ${ }^{* \dagger}$ & $270 \pm 282(\mathrm{n}=4)$ & $75 \pm 149(\mathrm{n}=10)$ \\
\hline CSF $\operatorname{IgG}^{* \dagger}$ & $8.9 \pm 4.8(\mathrm{n}=4)$ & $9.4 \pm 6.1(\mathrm{n}=10)$ \\
\hline expanded disability status scale (EDSS) ${ }^{*}$ & $6.8 \pm 1.4$ & $5.1 \pm 3.0$ \\
\hline $\begin{array}{l}\text { Attack frequency }{ }^{*} \\
\text { (per year) }\end{array}$ & $1.86 \pm 0.77$ & $1.72 \pm 1.28$ \\
\hline Number of autoantibodies ${ }^{*}$ & $2.80 \pm 1.60$ & $0.50 \pm 0.96$ \\
\hline
\end{tabular}

${ }^{*}$ mean $\pm \mathrm{SD} ;{ }^{\dagger} \mathrm{CSF}$ was obtained on the same day as the serum sample; ${ }^{\dagger} \mathrm{EDSS}$ were assessed when the condition of the patient was at its worst.

The EDSS scores correlated with the titer of the antimyelin IgG antibodies, MOG $\left(P=0.036, \mathrm{R}^{2}=0.37\right)$, $\operatorname{PLP}\left(P=0.042, \mathrm{R}^{2}=0.35\right)$ and $\operatorname{MBP}\left(P=0.036, \mathrm{R}^{2}=\right.$ 0.37) (Figure 1), and the score of all patients with high titers of antibodies against any of the myelin antigens was more than 4.5 (Table 1). However, there were no correlations between the EDSS scores and the titers of the following antibodies: anti-MOG $\operatorname{IgM}(P=0.76)$, anti-PLP $\operatorname{IgM}(P=0.78)$, anti-MBP $\operatorname{IgM}(P=0.75)$, antiMOG $\operatorname{IgG}(P=0.57)$, anti-PLP $\operatorname{IgG}(P=0.68)$, and anti$\operatorname{MBP}(P=0.45)$.

\subsection{Number of Autoantibodies Except for Anti-AQP4 and Anti-Myelin Antibodies}

The presence of other autoantibodies was examined in the sera of all patients. The antibodies examined were rheumatoid factor, anti-nuclear antibody, anti-Ro/SS-A antibody, anti-scleroderma-70 antibody, anti-cardiolipin antibody, lupus anticoagulant, thyroid stimulating antibody, anti-thyroid peroxidase antibody, and anti-thyroglobulin antibody. In the negative group, 1 patient had a complication of Sjögren syndrome. In the positive group, 1 patient exhibited anti-phospholipid antibody syndrome (APS), another had subclinical autoimmune thyroidism, and a third patient had a family history of systemic lupus erythematosus (SLE). The patients in the anti-myelin antibody-positive group presented with other autoantibodies more frequently than the negative group patients and this difference was significant (mean \pm SD, $2.80 \pm$ 1.60 versus $0.50 \pm 0.96, P=0.037$ with Welch's $t$ test).

\section{DISCUSSIONS}

In this retrospective case-control study, we found that the EDSS scores showed a correlation with the titer of the anti-myelin antibodies and an increase in the frequen- cy of anti-myelin antibodies in patients with NMO. ELISA was chosen to determine the presence of IgM and IgG anti-myelin antibodies since it is easily performed and can quantitatively analyze the titer of antibodies as OD values. All of the patients who were positive for anti-myelin antibodies had NMO or NMO spectrum diseases; none of the patients with conventional MS were positive for anti-myelin antibodies.

This frequency was lower than that found of a prior study that used ELISA to assess the serum level of antiMOG IgG (rh-MOG 1 - 125) in patients with MS. IgG MOG seropositivity was reported in $22 \%$ of patients with MS by Pittock, et al. and in 33\% of patients with MS by Wang, et al. [7,8]. This discrepancy may be due to the MOG-antigen used to coat the ELISA plate. For example, MOG-IgG (1 - 125) might be highly sensitive as an antigen compared to the MOG peptide (35 - 55). Another possible factor is the time-course used to determine the presence of anti-MOG antibodies since some patients who tested seropositive for MOG antibodies became seronegative during the follow-up period. O'Connor, et al. revealed that only the anti-MOG antibodies found in MS lesions had a high affinity for their antigens, whereas serum and CSF antibodies to MOG were low-affinity antibodies [12]. This means that the particular MOG antibodies detected could relate to the patterns of demyelination observed in MS.

Several studies have focused on the prognostic value of anti-MOG in the progression of MS in patients with clinical isolated syndrome (CIS). Berger, et al. reported that $\operatorname{IgM}$ anti-MOG antibodies and MBP antibodies, measured by Western blot, were predictors of MS development in a cohort of 103 patients with CIS [13]. Furthermore, these authors found that healthy young adults with detectable serum levels of IgG anti-MOG antibodies had an increased risk of developing MS. The predictive 

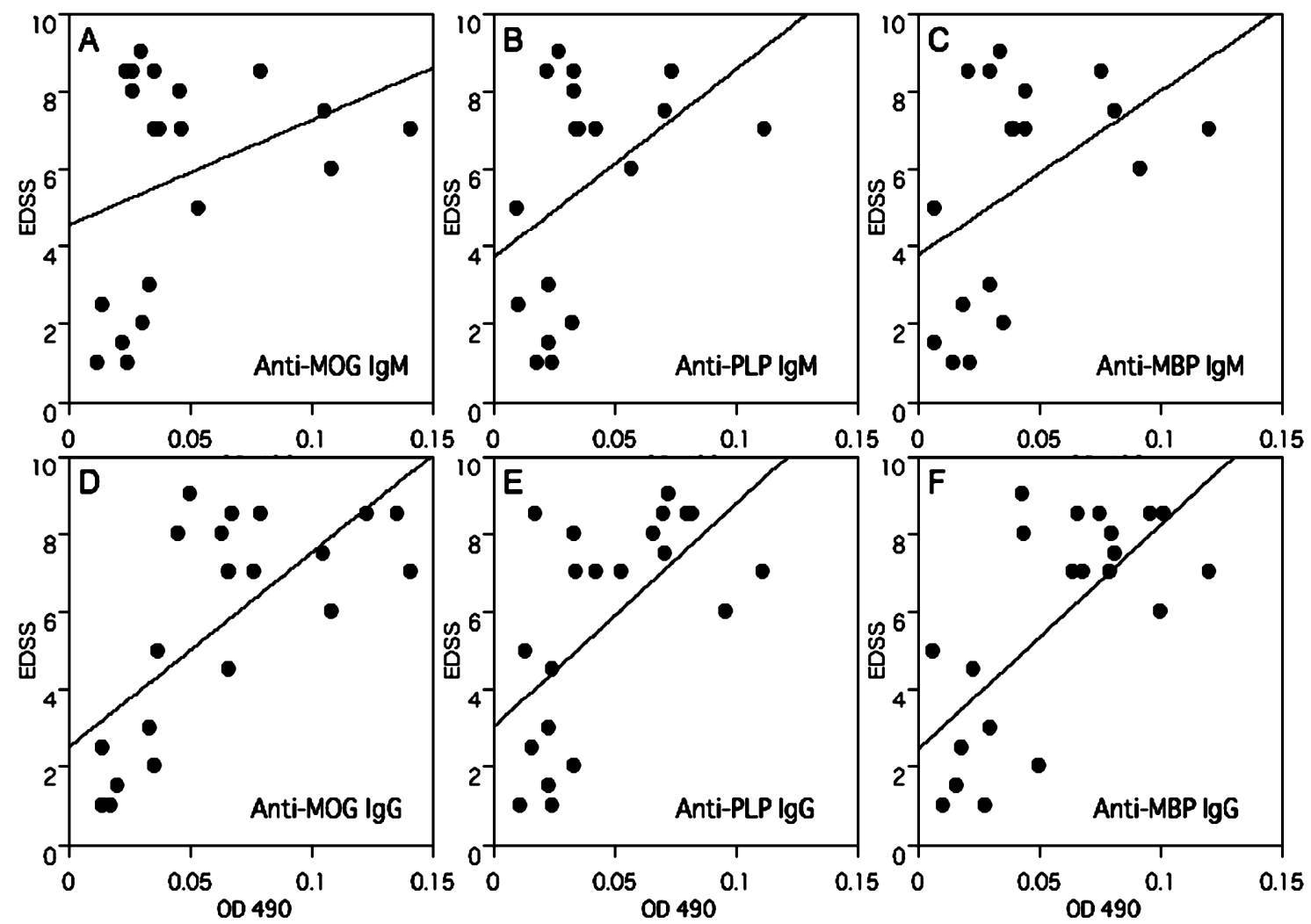

Figure 1. Correlation of the expanded disability status scale (EDSS) scores and the titers of autoantibodies to myelin antigens. The EDSS scores correlated with the titers of the following antibodies: anti-MOG $\operatorname{IgG}\left(P=0.036, \mathrm{R}^{2}=0.37\right)$, anti-PLP $\operatorname{IgG}\left(P=0.042, \mathrm{R}^{2}=0.35\right)$ and anti-MBP $\operatorname{IgG}\left(P=0.036, \mathrm{R}^{2}=0.37\right)$. There were no correlations between the EDSS scores and the titers of the following antibodies: anti-MOG $\operatorname{IgM}(P=0.76)$, anti-PLP $\operatorname{IgM}(P=0.78)$, anti-MBP $\operatorname{IgM}(P=0.75)$, anti-MOG $\operatorname{IgG}(P=0.57)$, anti-PLP $\operatorname{IgG}(P=0.68)$, and anti-MBP $(P=0.45)$. All patients with high titers of antibodies against myelin antigens scored more than 4.5 on the EDSS.

role of anti-myelin antibodies has been addressed in several different studies with correlations ranging from highly significant $[14]$ to partially significant $[15,16]$ to not significant $[17,18]$. The different results might reflect discrepancies in study designs and/or study populations.

In patients with NMO, autoantibody responses to the myelin antigens analyzed in this study by ELISA have previously been reported. All patients showed a positive and predominant anti-MOG response rather than an antiMBP autoantibody response [19]. It has been speculated that anti-MOG antibodies might be involved in the demyelinating process in NMO. To study immunologic alterations, Correale, et al. analyzed anti-MOG positive mononuclear cells in peripheral blood and CSF using ELISPOT assays. Their study found that MOG-specific responses in CSF showed significant increases in IgGand IgM-secreting cells in patients with NMO compared to patients with MS and healthy subjects [20]. These findings are in agreement with observations indicating more severe clinical manifestations in patients with NMO compared to those with MS. In the blood and CSF of patients with NMO, the mean number of IL-6-secret- ing cells was higher compared to that of MS and healthy control subjects. A strong correlation between the number of cells secreting anti-MOG antibodies and antigenspecific IL-6 synthesis was observed in CSF collected exclusively from patients with NMO [20].

Alves-Leon, et al. used ELISA to assess the production of $\operatorname{IgG}$ and IgA antibodies to antigens of MBP, PLP (95 - 116), and MOG (92 - 106) in 28 patients with NMO and healthy matched controls. Patients with NMO had significantly higher levels of IgGs to MOG $(P<0.0001)$, PLP $(P=0.0002)$, and MBP $(P<0.0001)$ compared to healthy controls [21]. Those authors suggested that the high expression of MOG (92 - 106), PLP (95 - 116), and MBP autoantibodies in patients with NMO could be a marker for the prognosis, recurrence, and/or severity of the disease.

In our study, the EDSS correlated with the titer of the anti-myelin antibodies. Furthermore, all patients with high titers of antibodies against myelin antigens scored higher than 4.5 points on the EDSS. These results could reflect severe inflammation of the central nervous system and poor prognoses in patients with anti-myelin antibo- 
dies. NMO is often associated with several autoantibodies, as well as anti-AQP4 antibodies, and these are also seen in other autoimmune diseases, especially autoimmune thyroidism, SLE, Sjögren syndrome, and APS [22, 23]. The overlap presence of these antibodies with other autoimmune diseases is considered a factor in the prediction of poor prognoses and/or poor responses to treatment, as reported in patients with NMO overlapping with Sjögren syndrome.

Our study revealed that the patients in the anti-myelin antibody-positive group presented with other autoantibodies more frequently than the negative group patients. In the positive group, 2 of 5 patients overlapped with other autoimmune diseases, while 1 patient had a family history of severe SLE.

We could clearly detect an association between the presence of these antibodies and the severity and clinical features of the disease. Our study also revealed increased EDSS scores in patients with high titers of anti-myelin antibodies. As reported in pathological and CSF studies, the demyelination that occurs subsequent to astrocytic damage in NMO is considered to be more severe than that observed in MS [24]. In addition, a recent study reported that anti-AQP4 antibodies damage oligodendrocytes through an excitotoxic mechanism following the disruption of glutamate homeostasis in astrocytes in vitro [25]. These findings allowed us to hypothesize that myelin antibodies were produced subsequent to the leakage of myelin antigens due to massive inflammation within the central nervous system.

In conclusion, anti-myelin antibodies in sera, as well as other autoantibodies and overlapping autoimmune diseases, may be useful markers for predicting severe clinical courses or poor prognoses in patients with NMO.

\section{ACKNOWLEDGEMENTS}

This work was supported in part by the Ministry of Health, Labor and Welfare of Japan (Health and Labor Sciences Research Grants; Research on intractable diseases, H23-017).

\section{REFERENCES}

[1] Wingerchuk, D.M. and Weinshenker, B.G. (2003) Neuromyelitis optica: Clinical predictors of a relapsing course and survival. Neurology, 60, 848-853. doi:10.1212/01.WNL.0000049912.02954.2C

[2] Carroll, W.M. and Fujihara, K. (2010) Neuromyelitis optica. Current Treatment Options in Neurology, 12, 244255. doi:10.1007/s11940-010-0071-Z

[3] Marignier, R., Nicolle, A., Watrin, C., et al. (2010) Oligodendrocytes are damaged by neuromyelitis optica immunoglobulin G via astrocyte injury. Brain, 133, 2578-2591. doi:10.1093/brain/awq177

[4] Sospedra, M. and Martin, R. (2005) Immunology of mul- tiple sclerosis. Annual Reviews of Immunology, 23, 683747.doi:10.1146/annurev.immunol.23.021704.115707

[5] Kerlero de Rosbo, N., Honegger, P., Lassmann, H., et al. (1990) Demyelination induced in aggregating brain cell cultures by a monoclonal antibody against myelin/oligodendrocyte glycoprotein. Journal of Neurochemistry, 55, 583-587. doi:10.1111/j.1471-4159.1990.tb04173.x

[6] Stefferl, A., Brehm, U., Storch, M., et al. (1999) Myelin oligodendrocyte glycoprotein induces experimental autoimmune encephalomyelitis in the resistant. Journal of Immunology, 163, 40-49.

[7] Pittock, S.J., Reindl, M., Achenbach, S., et al. (2007) Myelin oligodendrocyte glycoprotein antibodies in pathologically proven multiple sclerosis: Frequency, stability and clinicopathologic correlations. Multiple Sclerosis Journal, 13, 7-16. doi:10.1177/1352458506072189

[8] Wang, H., Munger, K.L., Reindl, M., et al. (2008) Myelin oligodendrocyte glycoprotein antibodies and multiple sclerosis in healthy young adults. Neurology, 71, 11421146.

[9] Kezuka, T., Usui, Y., Yamakawa, N., et al. (2012) Relationship between NMO-antibody and anti-MOG antibody in optic neuritis. Journal of Neuro-Ophthalmology, 32, 107-110. doi:10.1097/WNO.0b013e31823c9b6c

[10] Wingerchuk, D.M., Lennon, V.A., Pittock, S.J., et al. (2006) Revised diagnostic criteria for neuromyelitis optica. $\mathrm{Neu}$ rology, 66, 1485-1489. doi:10.1212/01.wnl.0000216139.44259.74

[11] Johns T.G. and Bernard C.C. (1999) The structure and function of myelin oligodendrocyte glycolprotein. Journal of Neurochemistry, 72, 1-9. doi:10.1111/j.1471-4159.1999.tb11579.x

[12] Khalis, M., Reindl, M., Lutterotti, A., et al. (2006) Epitope specificity of serum antibodies directed against the extracellular domain of myelin oligodendrocyte glycolprotein: Influence of relapses and immunomodulatory treatments. Journal of Neuroimmunology, 174, 147-156.

[13] Berger, T., Rubner, P., Schautzer, F., et al. (2003) Antimyelin antibodies as a predictor of clinically definitive multiple sclerosis after a first demyelinating event. The New England Journal of Medicine, 349, 139-145. doi:10.1056/NEJMoa022328

[14] Tomassini, V., De Giglio, L., Reindl, M., et al. (2007) Anti-myelin antibodies predict the clinical outcome after a first episode suggestive of MS. Multiple Sclerosis Journal, 13, 1086-1094. doi:10.1177/1352458507077622

[15] Rauer, S., Euler, B., Reindl, M. and Berger, T. (2006) Antimyelin antibodies and the risk of relapse in patients with a primary demyelinating event. Journal of Neurology, Neurosurgery \& Psychiatry, 77, 739-742. doi:10.1136/jnnp.2005.077784

[16] Kuhle, J., Pohl, C., Mehling, M., et al. (2007) Lack of association between antimyelin antibodies and progresssion to multiple sclerosis. The New England Journal of Medicine, 356, 371-378. doi:10.1056/NEJMoa063602

[17] Lim, E.T., Berger, T., Reindl, M., et al. (2005) Anti-myelin antibodies do not allow earlier diagnosis of multiple sclerosis. Multiple Sclerosis, 11, 492-494. 
[18] Pelayo, R., Tintoré, M., Montalban, X., et al. (2007) Antimyelin antibodies with no progression to multiple sclerosis. The New England Journal of Medicine, 356, 426-428. doi:10.1056/NEJMc062467

[19] Haase, C.G. and Schmidt, S. (2001) Detection of brainspecific autoantibodies to myelin oligodendrocyte glycolprotein, $\mathrm{S} 100 \beta$ and myelin basic protein in patients with Devic's neuromyelitis optica. Neuroscience Letters, 307, 131-133. doi:10.1016/S0304-3940(01)01949-8

[20] Correale, J. and Fiol, M. (2004) Activation of humoral immunity and eosinophils in neuromyelitis optica. Neurology, 63, 2363-2370. doi:10.1212/01.WNL.0000148481.80152.BF

[21] Alves-Leon, S.V., Pimentel, M.L., Sant'Anna, G., et al. (2008) Immune system markers of neuroinflammation in patients with clinical diagnose of neuromyelitis optica. Arquivos de Neuro-Psiquiatr, 66, 678-684. doi:10.1590/S0004-282X2008000500013
[22] Kahlenberg, J.M. (2011) Neuromyelitis optica spectrum disorder as an initial presentation of primary Sjögren's syndrome. Seminars in Arthritis and Rheumatism, 40, 343-348. doi:10.1016/j.semarthrit.2010.05.005

[23] Pittock, S.J., Lennon, V.A., De Seze, J., et al. (2008) Neuromyelitis optica and non-organ-specific autoimmunity. Archives of Neurology, 65, 78-83. doi:10.1001/archneurol.2007.17

[24] Takano, R., Misu, T., Takahashi, T., et al. (2010) Astrocytic damage is far more severe than demyelination in NMO. Neurology, 75, 208-216. doi:10.1212/WNL.0b013e3181e2414b

[25] Marignier, R., Giraudon, P., Vukusic, S., et al. (2010) Anti-aquaporin-4 antibodies in Devic's neuromyelitis optica: Therapeutic implications. Therapeutic Advances in Neurological Disorders, 3, 311-321. doi: $10.1177 / 1756285610382478$ 